2. Lee Lorch and Peter Szego, Higher monotonicity properties of certain SturmLiouville functions, Acta Math. 109 (1963), 55-73.

3. - Higher monotonicity properties of certain Sturm-Liouville functions. II, Bull. Acad. Polon. Sci. Sér. Sci. Math. Astronom. Phys. 11(1963), 455-457.

4. G. Szegö and P. Turán, On the monotone convergence of certain Riemann sums, Publ. Math. Debrecen. 8 (1961), 326-335.

5. P. Turán, On the zeros of the polynomials of Legendre, Casopis Pěst. Mat. 75 (1950), 113-122.

6. G. N. Watson, A treatise on the theory of Bessel functions, 2nd ed., Cambridge, England, 1944.

UNIVERSity OF Alberta, Edmonton AND

University of Santa Clara

\title{
COMPLETELY WELL-POSED PROBLEMS FOR NONLINEAR DIFFERENTIAL EQUATIONS
}

\author{
M. D. GEORGE ${ }^{1}$
}

1. Well-posed and completely well-posed problems for linear partial differential equations have been discussed by Hormander [2] and more recently and more generally by Browder [1]. Roughly speaking, if $L$ is a differential operator in a Banach space $X$, the problem of finding a solution of $L u=f, f \in X$, is said to be (completely) well-posed if the range of $L$ is $X$ and if in addition $L^{-1}$ exists and is (completely) continuous. In both papers, sufficient conditions are given for the existence of well-posed and completely well-posed problems for formal differential operators.

In this paper we are interested in the effect on a completely wellposed problem of a nonlinear perturbation of the operator $L$. In particular, we will show (Theorem 3) that under certain conditions a completely well-posed problem for a differential operator $L$ remains completely well-posed for $L+A$, where $A$ is a nonlinear transformation in $X$. Combining this result with theorems in [1], conditions guaranteeing the existence of completely well-posed problems for perturbed differential operators can be derived. One such result is given in Theorem 4 for the case $X=L^{2}$.

2. Let $X$ be a Banach space, $T$ a transformation with domain $D(T) \subset X$ and range $R(T) \subset X$. The transformations here are not assumed to be linear unless it is so stated.

Received by the editors November 5, 1962.

1 This research was supported by the Air Force Office of Scientific Research. 
Definition. The transformation $T$ is said to be asymptotic to zero if $D(T)=X$ and

$$
\lim _{\|u\| \rightarrow \infty} \frac{\|T u\|}{\|u\|}=0 .
$$

This definition is due to Krasnoselskil and the following theorem, referred to in [3], to Dubrovskir, who used it in treating nonlinear integral equations. A proof of this theorem is included here for the convenience of the reader.

THEOREM 1. If $T$ is completely continuous and asymptotic to zero, then $R(I+T)=X$.

Proof. Let $f$ be an arbitrary element of $X$. To prove that $u+T u=f$ has a solution, it suffices to prove that the transformation $S$ defined by $S u=f-T u$ has a fixed point. Noting that $S$ is completely continuous since $T$ is, we need only show, using the Schauder theorem, that $S$ maps some closed sphere of $X$ into itself.

Let

$$
B_{r}(f)=\{v \in X \mid\|v-f\| \leqq r\}
$$

and suppose that for each integer $n>0$, the set $S B_{n}(f)$ contains an element $S u_{n}$ not in $B_{n}(f)$. Then the sequence $\left\{u_{n}\right\}$ has the property that $\left\|u_{n}-f\right\| \leqq n$, while $\left\|S u_{n}-f\right\|=\left\|T u_{n}\right\|>n$. Since $T$ is completely continuous and the sequence $\left\{T u_{n}\right\}$ is unbounded, $\left\{u_{n}\right\}$ is also unbounded. On the other hand $\left\|u_{n}\right\| \leqq\|f\|+n$, so that

$$
\frac{\left\|T u_{n}\right\|}{\left\|u_{n}\right\|}>\frac{n}{\|f\|+n} \text {. }
$$

But this contradicts the assumption that $T$ is asymptotic to zero, since for such an operator $\|T u\| /\|u\|$ cannot be bounded away from zero as $u$ ranges over an unbounded set. Consequently, $S$ maps some $B_{n}(f)$ into itself, completing the proof.

The main result of this section is:

THEOREM 2. Let $L$ be a linear transformation, not necessarily bounded, with domain $D(L) \subset X$ and $R(L)=X$, and suppose $L$ has a completely continuous inverse. Let $A$ be bounded, continuous, and asymptotic to zero. Then $R(L+A)=X$.

The proof will follow easily from Theorem 1 with the aid of the following lemma.

Lemma. If $D(A)=D(K)=X$, and $K$ is linear and bounded while $A$ 
is bounded and asymptotic to zero, then $A K$ is asymptotic to zero.

Proof. It will be convenient to introduce some additional notation. Since $A$ is bounded, there is for each $r \geqq 0$ a number $s \geqq 0$ such that $A B_{r}(0) \subset B_{s}(0)$, using the notation introduced in (1). Denote by $M(r)$ the greatest lower bound of the set of such $s$. Also, since $A$ is asymptotic to zero, there is a real non-negative function $P(\epsilon)$ defined for $\epsilon>0$ such that $\|A v\|<\epsilon\|v\|$ whenever $\|v\|>P(\epsilon)$. Given $\epsilon>0$, set $\rho=P(\epsilon /\|K\|)$ (if $K=0$ the lemma is trivial) and let $u$ be any element of $X$ such that $\epsilon\|u\|>M(\rho)$. There are two cases to consider, according as $\|K u\|>\rho$ or $\|K u\| \leqq \rho$. In the first case,

$$
\frac{\|A K u\|}{\|u\|}=\frac{\|A K u\|}{\|K u\|} \frac{\|K u\|}{\|u\|} \leqq \frac{\|A K u\|}{\|K u\|}\|K\|<\frac{\epsilon}{\|K\|}\|K\|=\epsilon
$$

while in the second case

$$
\frac{\|A K u\|}{\|u\|}<\frac{M(\rho)}{M(\rho) / \epsilon}=\epsilon
$$

so that in any event, $\|A K u\|<\epsilon\|u\|$ for $\|u\|$ sufficiently large.

Proof of Theorem 2. Since $D(L+A)=D(L),(L+A) L^{-1}$ $=I+A L^{-1}=I+T$ is everywhere defined and from the lemma, $T$ is asymptotic to zero. Furthermore, since $A$ is continuous and $L^{-1}$ completely continuous, $T$ is completely continuous. Thus $R(I+T)=X$ by Theorem 1 and since $R(L)=X$ by hypothesis, it follows that $R(L+A)=R((I+T) L)=X$.

It should perhaps be noted here that Theorem 1 remains true under weaker hypotheses. For example, as is clear from the proof, one need only assume that for $\|u\|$ sufficiently large, $\|T u\| \leqq c\|u\|$ for some $c<1$. However, if $A$ has only this weaker property, $T=A L^{-1}$ need not have, unless some restriction is made on $\left\|L^{-1}\right\|$.

3. Let $K$ be any transformation in $X$. The problem of finding a solution $u \in D(K)$ of $K u=f, f \in X$, is said to be (completely) well-posed if $R(K)=X$ and $K$ has a (completely) continuous inverse. In this section we wish to consider the case in which $K$ is a differential operator and $X$ is a complex $L^{p}$ space, $1 \leqq p<\infty$. The description which follows is admittedly brief; full details can be found in [1].

Let $G$ be a bounded, open subset of Euclidean $n$-space, $n \geqq 1$. In now standard notation, we denote by

$$
P=\sum_{|\alpha| \leq m} a_{\alpha}(x) D^{\alpha}
$$

a linear differential operator of order $m$, with coefficients $a_{\alpha}(x)$ com- 
plex-valued functions on $G$, and by $P^{\prime}$, defined by

$$
P^{\prime} u=\sum_{|\alpha| \leq m} D^{\alpha}\left(\bar{a}_{\alpha}(x) u\right)
$$

its formal adjoint. We first consider $P$ and $P^{\prime}$ defined on $C_{0}^{\infty}(G)$, the infinitely-differentiable complex functions with compact support in $G$, and then close them as operators in $L^{p}(G)$ and $L^{p^{\prime}}(G)$ respectively, where $p^{\prime}=p /(p-1)$. These new operators will be denoted by $P_{0}$ and $P_{0}^{*} . P_{0}$ and $P_{1}$, the restricted adjoint of $P_{0}^{*}$, are called the minimal and maximal operators associated with the formal differential operator $P$. Let $L$ be a closed linear operator with $P_{0} \subseteq L \subseteq P_{1}$. If $R(L)$ $=L^{p}(G)$ and $L$ has a (completely) continuous inverse, then $L$ is said to be a (completely) solvable realization of the pair $\left(P_{0}, P_{0}^{*}\right)$ and the problem $L u=f$ is then (completely) well-posed.

THEOREM 3. Let $g(x, z)$ be a complex-valued function defined and uniformly continuous for $x \in G$, all complex $z$, such that

$$
|g(x, z)| \leqq c_{1}|z|^{a}+c_{2},
$$

where $c_{1}, c_{2}$, and a are non-negative constants and $a<1$. Denote by $A$ the operator in $L^{p}(G)$ defined by $A u(x)=g(x, u(x))$. If $L$ is a competely solvable realization of the pair $\left(P_{0}, P_{0}^{*}\right)$, then the problem $(L+A) u=f$, $f \in L^{p}(G)$, is completely well-posed provided $L+A$ has a continuous inverse.

Proof. The conditions imposed on $g(x, z)$ insure that $A$ is a bounded and continuous operator defined on $L^{p}(G)$. (Actually, less will suffice to give the same result. References to papers giving results along this line can be found in [3].) That $A$ is asymptotic to zero follows easily from (2) and hence $R(L+A)=L^{p}(G)$ from Theorem 2. Since $L+A$ has a continuous inverse by hypothesis, it remains only to show that in fact $(L+A)^{-1}$ is completely continuous. In view of the complete continuity of $L^{-1}$, it suffices to show that if $\left\{u_{n}\right\}$ is any sequence of elements in $D(L)=D(L+A)$ such that the set $\left\{(L+A) u_{n}\right\}$ is bounded, then the set $\left\{L u_{n}\right\}$ is also bounded. Suppose then that $\left\|(L+A) u_{n}\right\|<M$ while $\left\{L u_{n}\right\}$ is unbounded. Eliminating those $n$ 's for which $L u_{n}=0$ and setting $v_{n}=L u_{n}$ for the remainder, we have $\left\|A L^{-1} v_{n}\right\|=\left\|A u_{n}\right\| \geqq\left\|L u_{n}\right\|-M=\left\|v_{n}\right\|-M$, so that

$$
\frac{\left\|A L^{-1} v_{n}\right\|}{\left\|v_{n}\right\|} \geqq 1-\frac{M}{\left\|v_{n}\right\|} .
$$

But, as in the proof of Theorem 1, this last inequality is impossible 
since $A L^{-1}$ is asymptotic to zero. Thus $\left\{L u_{n}\right\}$ is a bounded set and so $\left\{u_{n}\right\}$ has a convergent subsequence.

It is clear that the theorem could be immediately generalized to transformations of the form $L+K A M$, where $A$ is as described in the theorem and $K$ and $M$ are linear and bounded. That $K A M$ is asymptotic to zero follows easily from the lemma.

Finally, a word may be said about the existence of completely wellposed problems for the formal differential operator $P+A$. By combining these results with theorems of [1] and [2], a number of results can be obtained. One example will suffice to illustrate the idea.

TheOREM 4. Let $p=2$ and let $A u=g(x, u(x))$, where $g(x, z)$ satisfies the conditions of Theorem 3. Suppose $P_{0}$ and $P_{0}^{*}$ have completely continuous inverses on their respective ranges. Then there exists a completely solvable realization $L$ of the pair $\left(P_{0}, P_{0}^{*}\right)$ and a number $c>0$ such that if $|g(x, u)-g(x, v)| \leqq c|u-v|$ for all complex $u$, $v$ and all $x$ in $G$, then the problem $(L+A) u=f, f \in L^{2}(G)$, is completely well-posed.

Proof. The existence of $L$ follows from Theorem 1.2 of [2]. (The corresponding theorem for reflexive Banach spaces can be found in [1].) An easy calculation shows that if $|g(x, u)-g(x, v)| \leqq c|u-v|$, where $c\left\|L^{-1}\right\|<1$, then for $u(x), v(x) \in D(L)=D(L+A)$,

$$
\|u-v\| \leqq\left\|L^{-1}\right\|\left(1-c\left\|L^{-1}\right\|\right)^{-1}\|(L+A) u-(L+A) v\| .
$$

Hence $L+A$ has a continuous inverse and the conclusion follows from Theorem 3.

Note that the constant $c$ can be estimated from the construction of $L^{-1}$ given in the proof of Theorem 1.2 in [2]. Hörmander also gives in [2] conditions which insure the complete continuity of $P_{0}^{-1}$ and $\left(P_{0}^{*}\right)^{-1}$.

\section{REFERENCES}

1. F. Browder, Functional analysis and partial differential equations. II, Math. Ann. 145 (1962), 81-226.

2. L. Hörmander, On the theory of general partial differential operators, Acta Math. 94 (1955), 161-248.

3. M. A. KrasnoselskiY, Some problems of nonlinear analysis, Uspehi Mat. Nauk 9 (1954), no. 3 (61), 57-114; English transl., Amer. Math. Soc. Transl. (2) 10 (1958), 345-409.

UNIVERSITY OF MisSOURI 